\title{
Symmetrical Thalamic Lesions in the Newborn: A Case Series
}

\author{
T. Pols ${ }^{1,2} \quad$ L.S. de Vries ${ }^{3} \quad$ A. Soltirovska Salamon ${ }^{4} \quad$ P.G.J. Nikkels ${ }^{5} \quad$ K.D. Lichtenbelt ${ }^{6}$ \\ S.M. Mulder-de Tollenaer ${ }^{1}$ G. van Wezel-Meijler ${ }^{1}$
}

${ }^{1}$ Department of Neonatology, Isala Women and Children's Hospital, Zwolle, The Netherlands

${ }^{2}$ Department of Neonatology, Maxima Medical Center, Veldhoven, The Netherlands

${ }^{3}$ Department of Neonatology, Wilhelmina Children's Hospital, University Medical Center Utrecht, Utrecht, The Netherlands

${ }^{4}$ Department of Neonatology, University Children's Hospital,

University Medical Center, Ljubljana, Slovenia

${ }^{5}$ Department of Pathology, University Medical Center Utrecht, Utrecht, The Netherlands

${ }^{6}$ Department of Medical Genetics, University Medical Center Utrecht, Utrecht, The Netherlands

Address for correspondence Gerda Meijler, MD, PhD, PediatricianNeonatologist, Department of Neonatology, V4.4, Isala Women and Children's Hospital, P.O. Box 10400, 8000 GK Zwolle, The Netherlands (e-mail: g.meijler@isala.nl).

Neuropediatrics 2019;50:152-159.

\begin{abstract}
Although bilateral injury to the thalami is often seen in (near)term infants with hypoxic ischemic encephalopathy (HIE), symmetrical thalamic lesions (STL) is a different, very rare condition, seen both in full-term and preterm infants often after an antenatal insult, although the history is not always clear. These lesions are usually first detected using cranial ultrasound (cUS). They may not always be seen on the first (admission) scan, but become apparent in the course of the 1st week after birth. Clinically, these infants present with hypo- or hypertonia, absence of sucking and swallowing reflexes,

\section{Keywords}

- symmetrical thalamic lesions

- newborn

- neuroimaging and they may have contractures and facial diplegia. Neuropathology commonly demonstrates a thalamic lesion with additional and variable involvement of basal ganglia and brainstem. The prognosis is very poor, the condition often leads to severe disabilities and/or death within the first years of life. The clinical course and neuroimaging findings of 13 patients with symmetrical thalamic lesions (STL) are reported.
\end{abstract}

\section{Introduction}

Symmetrical thalamic lesions (STL) were first described in 1962 by Rosales and Riggs. ${ }^{1}$ This condition has also been referred to as symmetrical thalamic calcifications (STC). ${ }^{2,3}$ STL is a specific neuropathological entity, often following an acute antenatal insult but the etiology is not always known and still a matter of debate. It differs from a postnatal (mostly unilateral) hemorrhage or bilateral ischemic injury in the thalamus following a severe perinatal hypoxic-ischemic event. ${ }^{4}$ It is thought that the vulnerability of the central gray matter to injury at term age is related to rapid myelination and

received

August 14, 2018

accepted after revision

February 8, 2019

published online

March 26, 2019

thus high metabolic demands in this region at that age. The first parts of the central gray matter to myelinate are the ventrolateral and subthalamic nuclei where myelination starts in the early preterm period. ${ }^{5}$ Histopathology in STL shows bilateral lesions with gliosis, neuronal mineralization, and loss of neurons, mostly involving the lateral part of the thalami. The histological abnormalities of the affected neurons do not differ from typical hypoxic-ischemic injury, and can be seen after severe hypoglycemia or a toxic insult as well. Sparing of other parts of the brain with a similar sensitivity to hypoxic-ischemic injury, might argue against a hypoxic-ischemic event.

(c) 2019 Georg Thieme Verlag KG Stuttgart · New York
DOI https://doi.org/ 10.1055/s-0039-1683864. ISSN 0174-304X. 
Because STL is uncommon and not always easy to recognize, we hereby present our case series including the clinical history and neuroimaging findings of 13 patients.

\section{Methods}

The clinical course and cranial ultrasound (cUS) and magnetic resonance imaging (MRI) findings of all 13 neonates with STL admitted between 1991 and 2017 to two level three neonatal intensive care units (NICUs) in the Netherlands and one NICU in Slovenia are presented and compared with available data in the literature. cUS was performed on admission and then repeated regularly until discharge home or death. MRI, using a 1.5 or 3 Tesla's magnet was performed in the neonatal period in 11 cases. One patient underwent a computed tomography (CT) scan and one had no MRI because the infant died before the MRI could be performed. The MRI protocol included at least T1- and T2weighted sequences and in more recent cases also diffusionand susceptibility-weighted imaging.

\section{Results}

\section{Patient Characteristics and Clinical Symptoms}

Polyhydramnios and reduced fetal movements were present in $4 / 13$ pregnancies. An episode of acute and/or recurrent severe fetal hypoxia was recorded in 9/13 pregnancies. Median gestational age of the neonates was $34^{6 / 7}$ weeks (range, $25^{6 / 7}-39^{6 / 7}$ weeks). There was a striking female preponderance (11/13). One patient was small for gestational age (SGA) and nine patients were born prematurely (gestational age $<37$ weeks). Low Apgar's scores ( $\leq 6$ at 5 minutes) were present in five. Five patients needed insufflation breaths and one (case 7) was intubated after 15 minutes because of lack of spontaneous respiration. Intubation and ventilation were subsequently required in 6/13 infants. Clinical symptoms included marked central hypotonia in all, followed by hypertonia in seven patients, contractures in four, failed neonatal hearing test in six (five automated auditory brainstem response [AABR]; two brainstem evoked response audiometry [BERA]; one patient failed both) and swallowing problems in all but one. Amplitude integrated electroencephalography (aEEG) recordings were performed in eight patients. In one of them seizure activity was demonstrated. Ten infants died, five in the neonatal period and five in the 1st year beyond the neonatal period. The three surviving infants had a severely abnormal neurological outcome at 12 to 48 months, with absent sucking and swallowing, lack of visual interaction, abnormal hearing, and hypertonia and contractures in all extremities.

\section{Neuroimaging Findings}

cUS showed abnormal, mostly progressive echogenicity in the thalami in all patients. In 6/13 cases this was seen on the first scan after birth and in the other cases within 1 week after birth. The most prominent abnormalities on the first cUS scan were seen in the most premature infants. In 5/13 cases the echogenicity increased further over time. We did not find a relationship between the severity of the cUS abnormalities and the clinical symptoms. In the 11 patients in whom MRI was performed, T1-weighted sequences showed increased signal intensity in the thalami in all. T2-weigthed images also showed signal abnormalities in the thalami, but this was less obvious than on the T1-weighted images. Diffusion weighted images showed no diffusion restriction, probably related to the long time period between the MRI and the presumed insult. When the MRI was performed or repeated at or beyond term equivalent age, delayed myelination was seen in the posterior limb of the internal capsule. There was no obvious other maturational delay. Additional abnormalities were seen in the white matter in two, the globus pallidus in another two, and the cerebellum in one infant.

\section{Histopathology Findings}

Autopsy was performed in four cases. Histology showed loss of neurons in the thalami with mild calcifications and iron staining of individual neurons in all of them. Case 9 showed extensive calcifications. In case 3 , there was massive loss of neurons in the thalami with some additional neuronal loss and gliosis in the hippocampus. In case 6 , the nuclei of the cranial nerves showed loss of neurons and iron staining of neurons ( - Fig. 1A and B). Case 7 showed abnormalities in both thalami with neuronal mineralization and involvement of the reticular formation. Also, both nuclei of the facial nerve were severely damaged. Cases 6 and 7 show some overlap with expanded Möbius's syndrome as described by Peleg et al. ${ }^{6}$

\section{Ophthalmological Findings}

An ophthalmological examination was done in $7 / 13$ patients and unremarkable in all.

- Table 1 provides clinical and neuroimaging data of the 13 patients. Below we describe details of the clinical presentation and neuroimaging findings of two of our recent patients.

\section{Case Description}

\section{Case 1}

The mother was admitted at $23^{5 / 7}$ weeks gestation because of recurrent vaginal blood loss due to placenta praevia, premature rupture of membranes, and severe oligohydramnios. Corticosteroids were administered to promote fetal lung maturation.

At $25^{6 / 7}$ weeks gestation an emergency caesarean section was performed because of acute, massive vaginal blood loss, and pronounced fetal distress. A girl was born with a birth weight of $700 \mathrm{~g}$. Apgar scores were 4, 6, and 8 at 1, 5, and 10 minutes, respectively. The arterial umbilical cord $\mathrm{pH}$ was 7.15 with a base excess of $10 \mathrm{mmol} / \mathrm{L}$. The first hemoglobin after birth was $8.7 \mathrm{mmol} / \mathrm{L}$ with a hematocrit of $0.41 \mathrm{~L} / \mathrm{L}$. On physical examination she had contractures of both elbows and her left knee, probably related to the oligohydramnios.

She initially received insufflation breaths, followed by nasal continuous positive airway pressure (CPAP) with supplemental oxygen and was transported to the NICU. She was intubated shortly after admission because of insufficient breathing efforts and respiratory failure due to pulmonary hypoplasia. 


\begin{tabular}{|c|c|c|c|c|c|c|c|c|c|c|c|c|c|c|c|c|c|c|}
\hline$\stackrel{m}{=}$ & \begin{tabular}{|l|} 
\\
$\vdots$ \\
$\vdots$ \\
$m$ \\
$m$
\end{tabular} & $\begin{array}{l}\stackrel{D}{\sim} \\
m \\
m\end{array}$ & $\frac{\frac{\infty}{0}}{m}$ & \begin{tabular}{|l|}
$\frac{0}{\tilde{T}}$ \\
$\tilde{E}$ \\
$\tilde{U}$ \\
\end{tabular} & $\ddot{\infty}$ & 9 & $\cong$ & + & + & + & 1 & + & $\supset$ & ++ & $+\vdots$ & 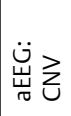 & 2 & 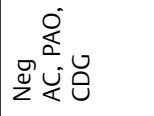 \\
\hline$\simeq$ & $\mid \begin{array}{l}1 \\
0 \\
\dot{m} \\
\dot{m}\end{array}$ & 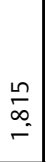 & $\frac{\pi}{6}$ & $\frac{0}{\frac{0}{n \pi}}$ & 0 & 气 & 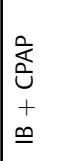 & + & + & + & & + & 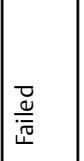 & + & + & $\hat{z}$ & z & 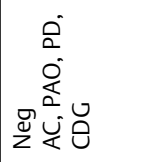 \\
\hline$=$ & 品 & $\begin{array}{l}0 \\
\stackrel{0}{m} \\
i\end{array}$ & $\frac{0}{\frac{0}{\infty}}$ & 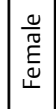 & a & 9 & z & + & + & + & 1 & + & $\frac{\overline{\ddot{\nu}}}{\overline{\overline{\tilde{w}}}}$ & ++ & + & $\frac{n}{z}$ & z & $\stackrel{\Xi}{z} \tilde{z}$ \\
\hline$\circ$ & $\mid \begin{array}{l}\hat{\bar{n}} \\
\mathrm{~m} \\
\end{array}$ & $\begin{array}{l}q \\
\vdots \\
0 \\
i\end{array}$ & $\frac{0}{m}$ & 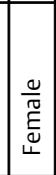 & a & 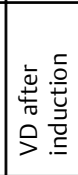 & 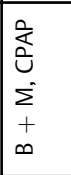 & 1 & + & + & 1 & + & $\supset$ & + & $+\sum_{a}^{\vec{n}}$ & $\begin{array}{l}z \\
\text { zu } \\
\text { u }\end{array}$ & 2 & 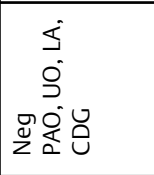 \\
\hline$\sigma$ & $\mid \begin{array}{c}\bar{\lambda} \\
\tilde{m} \\
m\end{array}$ & $\begin{array}{l}\stackrel{o}{o} \\
- \\
-\end{array}$ & $\begin{array}{l}\frac{0}{0} \\
\frac{0}{1}\end{array}$ & 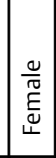 & 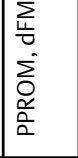 & 9 & 2 & + & 1 & + & 1 & $\supset$ & $\supset$ & + & $\hat{z}$ & 总 & 2 & $\stackrel{\Xi}{\tilde{z}} \tilde{z}$ \\
\hline$\infty$ & $\stackrel{\stackrel{n}{m}}{\mathrm{~m}}$ & $\Sigma$ & $\sum$ & 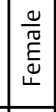 & 住 & $\supset$ & $\supset$ & + & $\supset$ & $\supset$ & 1 & $\supset$ & $\supset$ & + & $+\sum$ & $\begin{array}{l}u \\
\ddot{u} \\
\end{array}$ & 2 & $\supset$ \\
\hline$\wedge$ & 竞 & $\begin{array}{l}n \\
\tilde{o} \\
i \\
i\end{array}$ & $\frac{n}{N}$ & 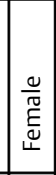 & $\sum_{i=0}^{0}$ & 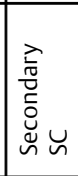 & 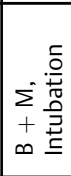 & + & + & $\supset$ & 1 & + & 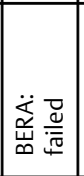 & ++ & + & 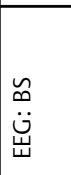 & 2 & 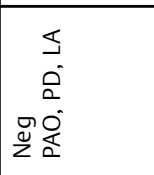 \\
\hline 0 & 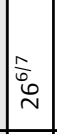 & \& & 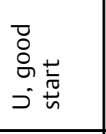 & 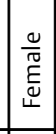 & 玹 & 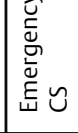 & z & + & 1 & $\supset$ & + & + & $\supset$ & ++ & $\begin{array}{l}+ \\
\ddot{U}\end{array}$ & $\hat{z}$ & 2 & 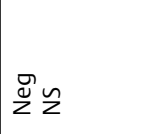 \\
\hline in & 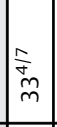 & $\begin{array}{l}0 \\
0 \\
\infty \\
\infty \\
- \\
\end{array}$ & $\frac{\sigma}{\infty}$ & 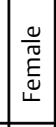 & 部 & 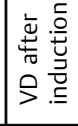 & z & 1 & 1 & $\supset$ & + & + & 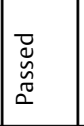 & ++ & 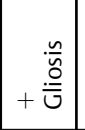 & $\frac{\hat{z}}{z}$ & z & $\frac{n}{z}$ \\
\hline$\sigma$ & $\mid \begin{array}{l}1 \\
\vdots \\
0 \\
m\end{array}$ & 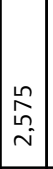 & $\frac{0}{\sigma}$ & \begin{tabular}{|l|}
$\frac{0}{\pi}$ \\
$\stackrel{\tilde{T}}{\tilde{E}}$ \\
$\stackrel{L}{L}$ \\
\end{tabular} & $\sum_{\text {任 }}$ & 9 & z & + & + & + & + & + & $\supset$ & + & 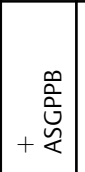 & $\begin{array}{l}\sum \\
\ddot{j} \\
\text { 㟧 }\end{array}$ & 2 & 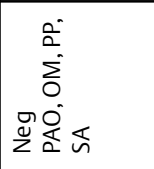 \\
\hline$m$ & \begin{tabular}{|c|} 
\\
$\substack{n \\
m \\
m}$ \\
\end{tabular} & \begin{tabular}{|c|} 
\\
$\infty$ \\
$\infty$ \\
$\sim$ \\
\end{tabular} & $\frac{9}{\infty}$ & 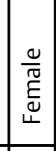 & 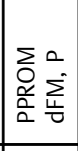 & 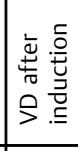 & 2 & + & + & + & 1 & + & 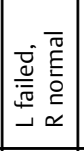 & + & $\begin{array}{r}0 \\
0 \\
\vdots \\
+\vdots \\
\end{array}$ & $\begin{array}{l}z \\
u \\
\ddot{u} \\
\text { u }\end{array}$ & 2 & 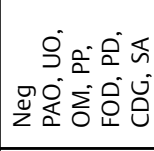 \\
\hline$N$ & 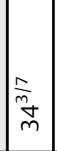 & 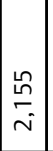 & $\frac{\frac{\infty}{\sigma}}{\frac{\sigma}{\gamma}}$ & $\frac{0}{\frac{0}{50}}$ & 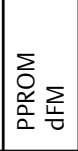 & 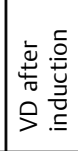 & $\supset$ & + & + & + & 1 & + & 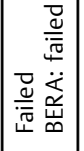 & ++ & + & $\begin{array}{l}\tilde{z} \\
\ddot{u} \\
\text { 㟧 }\end{array}$ & ₹ & ن \\
\hline- & 立 & ঃ & $\frac{\frac{\infty}{0}}{\frac{0}{\sigma}}$ & 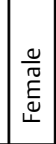 & 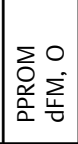 & 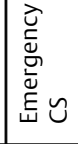 & $\begin{array}{l}\stackrel{0}{\mathbf{c}} \\
\text { Ù } \\
+ \\
\cong\end{array}$ & + & + & + & + & + & 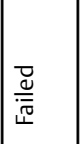 & + & $\begin{array}{r}\Sigma \\
\Delta \\
I \\
+\stackrel{I}{~}\end{array}$ & $\bar{z}$ & ₹ & 定 \\
\hline 岕 & \begin{tabular}{|l|}
$\frac{2}{3}$ \\
$\frac{5}{5}$
\end{tabular} & 이 & 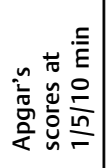 & $\stackrel{x}{\sim}$ & 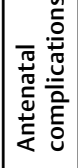 & 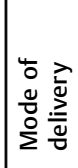 & 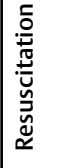 & 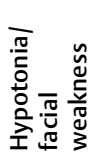 & . & 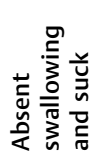 & 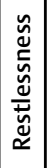 & 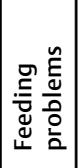 & 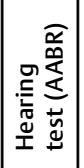 & 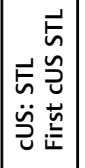 & 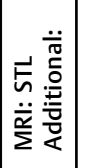 & 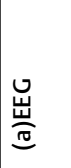 & 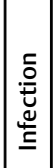 & 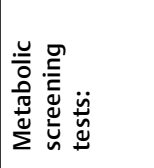 \\
\hline
\end{tabular}




\begin{tabular}{|c|c|c|c|c|c|c|c|c|}
\hline 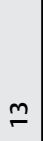 & 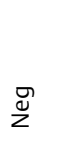 & $\hat{z}$ & + & + & & 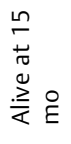 & & + \\
\hline$\simeq$ & $\frac{\hat{z}}{z}$ & 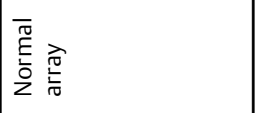 & +1 & & & 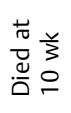 & & \\
\hline$F$ & $\frac{0}{z}$ & $\sum \frac{\vec{d}}{\frac{\pi}{0}}$ & +1 & + & 1 & 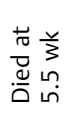 & & \\
\hline$\stackrel{\circ}{-}$ & $\hat{z}$ & 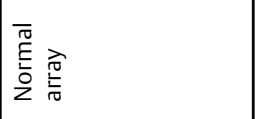 & 1 & 1 & & 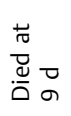 & & \\
\hline$\sigma$ & $\hat{z}$ & $\supset$ & 10 & 1 & & 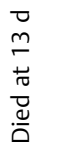 & + & \\
\hline$\infty$ & $\supset$ & $\supset$ & 11 & 1 & + & 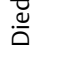 & & \\
\hline$\wedge$ & $\hat{z}$ & 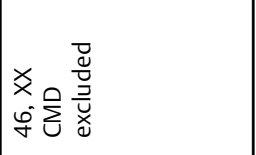 & 1 & 1 & & 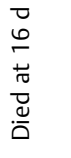 & + & \\
\hline 0 & $\hat{z}$ & $\supset$ & 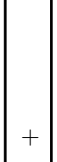 & + & 1 & 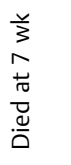 & + & \\
\hline in & $\hat{z}$ & $\hat{z}$ & 1 & + & 1 & 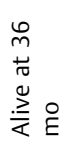 & & + \\
\hline$\nabla$ & $\stackrel{\widetilde{v}}{z}$ & 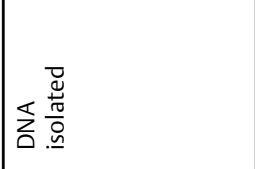 & 1 & 1 & & 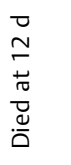 & & \\
\hline$m$ & $\supset$ & 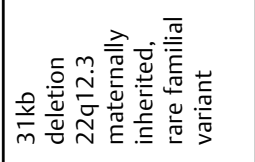 & + & + & & 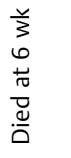 & + & \\
\hline$\sim$ & $\supset$ & 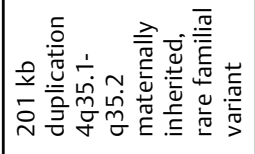 & + & + & + & 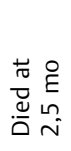 & & \\
\hline- & $\stackrel{\mathbb{v}}{z}$ & $\supset$ & + & + & 1 & 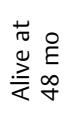 & & + \\
\hline 岕 & 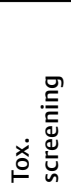 & 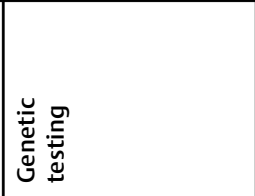 & in & Oे & 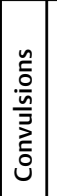 & $\begin{array}{l}\frac{7}{\pi} \\
\frac{d}{2} \\
\frac{d}{2} \\
\frac{2}{<}\end{array}$ & 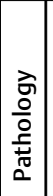 & 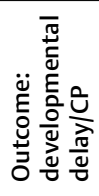 \\
\hline
\end{tabular}

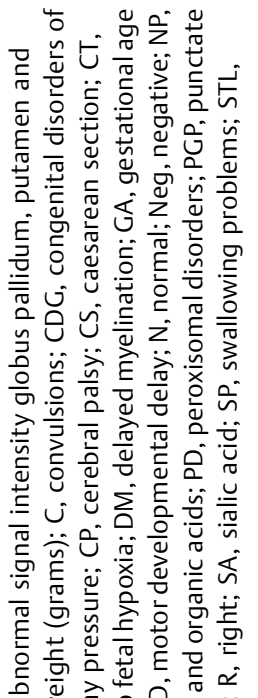

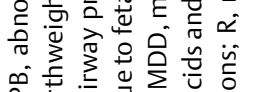

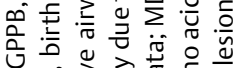

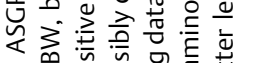

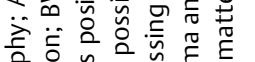

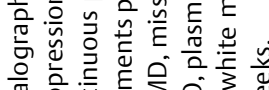

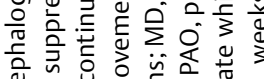

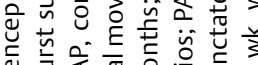

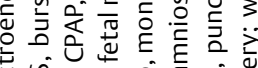

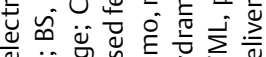

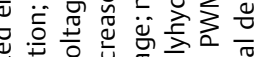

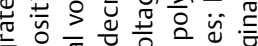

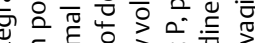

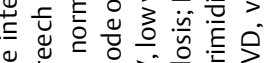

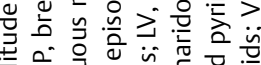

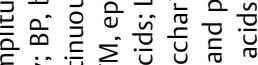

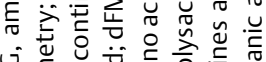

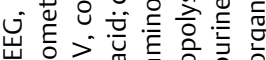

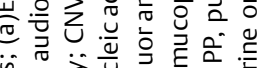

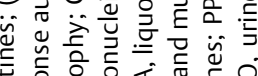

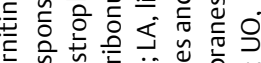

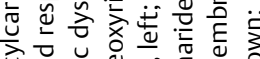

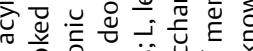

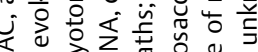
틀

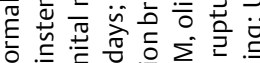

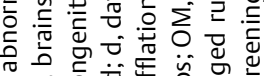

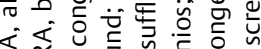
武岩 字总

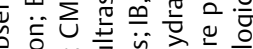
空

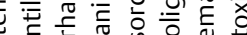
i)

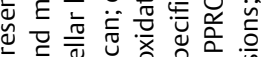
元 E

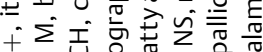

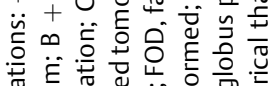

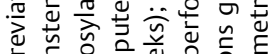

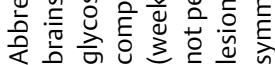




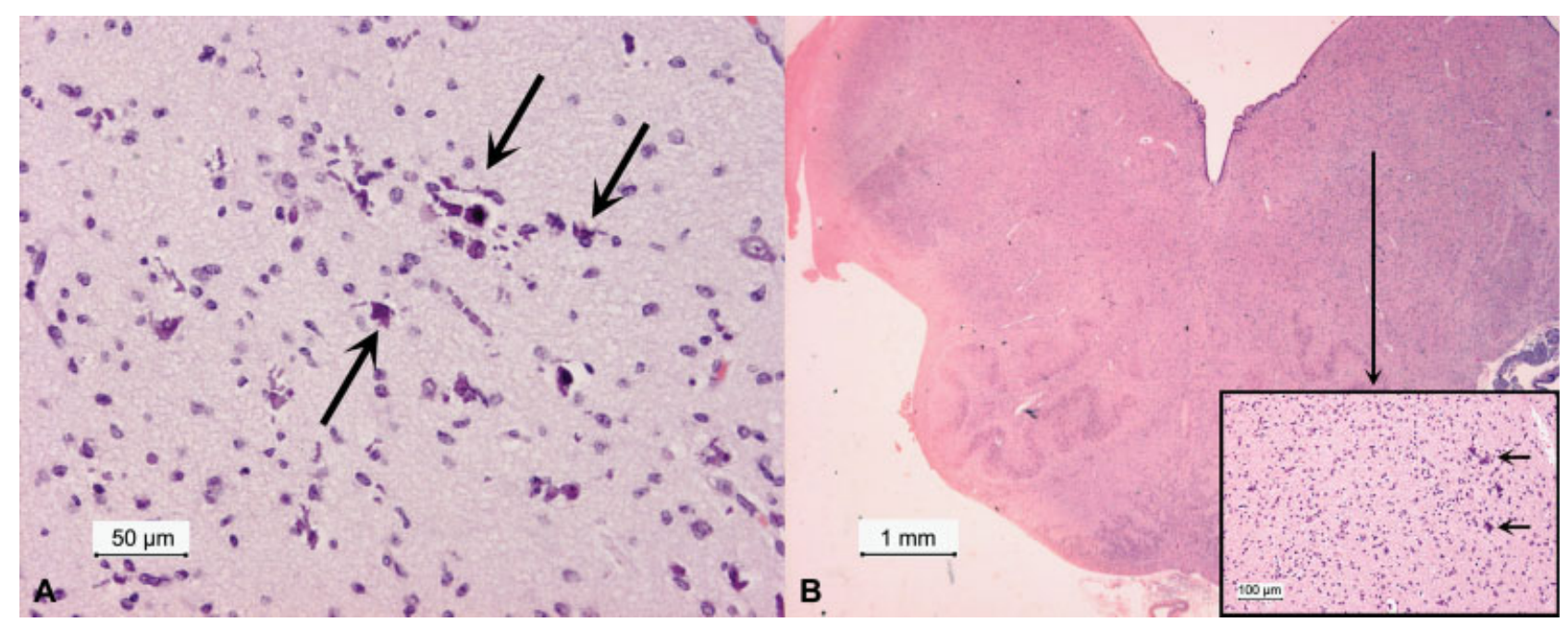

Fig. 1 Case 6. (A) High power view (H and E stain) of the thalamus with extensive loss of neurons and ferrugination of neurons (arrows). (B) Low power view of the brainstem with inferior olivary nucleus with normal aspect. Inset is high power view of the nuclei of the cranial nerves with loss of neurons and ferrugination of neurons (arrows). Published in color online. $\mathrm{H}$ and $\mathrm{E}$ stain, hematoxylin and eosin

Because of severe hypotension she received two fluid boluses and low dose dopamine $(5 \mu \mathrm{g} / \mathrm{kg} / \mathrm{min})$.

On the 1 st postnatal day she was uncomfortable and restless for which she was treated with a loading dose of phenobarbitone $(20 \mathrm{mg} / \mathrm{kg}$, intravenously). In addition, she received morphine (maximum $10 \mu \mathrm{g} / \mathrm{kg} / \mathrm{h}$ ) during the period of assisted ventilation.

The first two cUS scans, respectively performed a few hours after birth and on the 2nd day, did not show abnormalities. cUS on day 4 showed abnormal bilateral echogenicity of the basal ganglia and thalami (Fig. 2A).

Her further stay on the NICU was characterized by development of bronchopulmonary dysplasia and recurrent atelectasis for which long-term respiratory support (2.5 weeks) and eventually hydrocortisone administration was needed. She also suffered from recurrent infections. She failed the neonatal hearing test (AABR).

She had severe neurological symptoms, consisting of disturbed swallowing and sucking, abnormal tone, and hyperreflexia. These symptoms were ascribed to the deep gray matter abnormalities. Possible causes for the abnormalities seen on cUS were hypoxic-ischemic injury, kernicterus, congenital infection, hemorrhage, metabolic disease, sulfite oxidase deficiency, mitochondrial disorders, and maternal vitamin B12 deficiency. TORCHeS (Toxoplasmosis, Other, Rubella, Cytomegalovirus, Herpes) serology was negative. Extensive metabolic screening (including amino acids, organic acids, acylcarnitine, transferrin) was normal; sulfite oxidase deficiency and maternal vitamin B12 deficiency were also excluded. Kernicterus was very unlikely as bilirubin levels remained far below the exchange transfusion levels throughout the neonatal period (maximum $114 \mu \mathrm{mol} / \mathrm{L}$ ). In addition, the abnormalities were mainly located in the thalami and less in the basal ganglia. MRI of the brain, performed on postnatal day 17 at a postmenstrual age (PMA) of $28^{2 / 7}$ weeks showed only subtle abnormalities of the basal ganglia and thalami. The white matter appeared normal. There were two small hemorrhages in the cerebellum
(-Fig. 2B). Serial cUS examinations of the brain showed increasingly abnormal echogenicity of the basal ganglia and thalami (-Fig. 2C). Because severe neurological symptoms were present immediately after birth, the cord $\mathrm{pH}$ and base excess were not very low and the abnormalities in the thalami were much more severe on early cUS than MRI, hypoxicischemic brain injury after perinatal asphyxia seemed less likely and the diagnosis STL was made.

At term equivalent age (PMA 41 weeks) MRI was repeated (-Fig. 2D) and showed bilateral inhomogeneous signalintensity in the globus pallidus, putamen, ventrolateral thalamic nuclei, and hippocampi. The extracerebral fluid spaces were enlarged, related to white matter volume loss. There were multiple punctate hemorrhages in both cerebellar hemispheres and there was only sparse myelination of the posterior limb of the internal capsule. The cerebellar myelination was also delayed.

She was discharged home at the corrected age of 2 months and was readmitted several times because of respiratory infections. At the age of 4 years she has severe feeding and swallowing difficulties. She has developed cerebral palsy (GMFCS level IV) and a marked cognitive delay, but is showing some developmental progression with normal vision and hearing.

\section{Case 2}

A boy was born at $34^{3 / 7}$ weeks of gestation with a birthweight of 2,155 g (P50 Hindi-curve) and Apgar scores of 4, 6, and 8 at 1,5 , and 10 minutes respectively. He was the first child of nonconsanguineous Indian parents. Ten days before birth the mother felt reduced fetal movements.

The membranes ruptured at $34^{2 / 7}$ weeks of gestation. The amniotic fluid was meconium stained. Labor was induced and the mother received antibiotics. Labor was uneventful. The umbilical cord was very short without strangulation.

The infant had some dysmorphic features: hypertelorism, micrognathia, and low set ears. He was hypotonic, which resolved after a few hours. He had some cyanotic spells, 


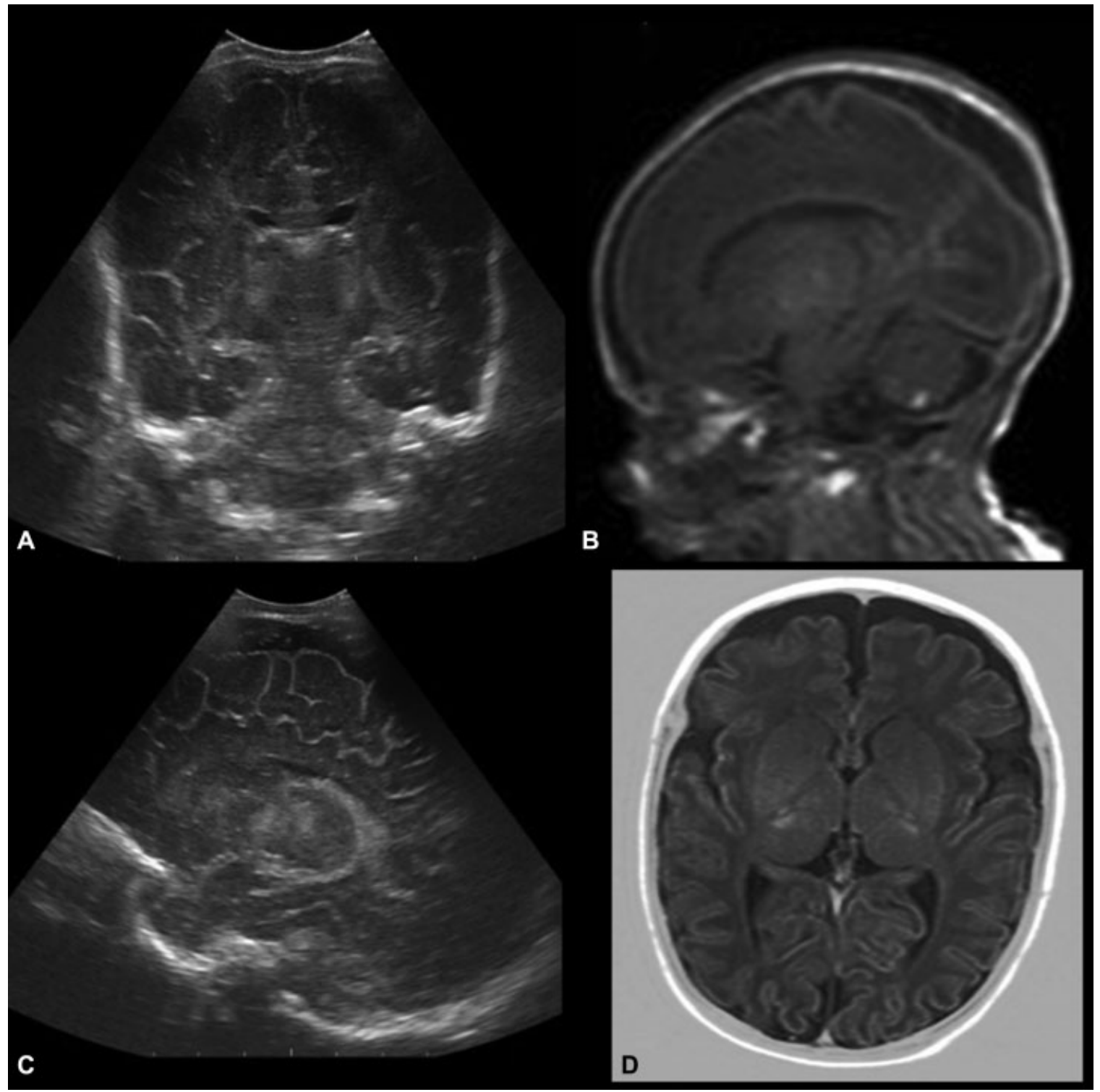

Fig. 2 Case 1. (A) Bilateral abnormal echogenicity of the basal ganglia and thalami on coronal cUS on postnatal day 4. (B) Subtle abnormalities of the basal ganglia and thalami and a small hemorrhage in the cerebellum on sagittal MRI T1-weighted sequence on day 17 . (C) Increasingly abnormal echogenicity of the basal ganglia and thalami on serial cUS examinations (parasagittal scan) of the brain. (D) Transverse MRI T1weighted sequence at term equivalent age at midventricular level shows small areas of increased signal intensity in the ventrolateral thalami and reduced thalamic volumes. cUS, cranial ultrasound; MRI, magnetic resonance imaging.

thought to be related to the prematurity and the micrognathia, for which an oropharyngeal airway was placed and he was started on caffeine. He had difficulties swallowing and was tube fed. After 24 hours he developed clinical seizures with head deviation toward the right side and tonic clonic movements of his right arm and leg. This was successfully treated with phenobarbitone. The clinical seizures were not confirmed on the aEEG.

Laboratory-examination revealed no signs of an underlying cause; hypoglycemia and electrolyte-disturbances were excluded. Metabolic tests (including amino acids, organic acids, acylcarnitine, and transferrin) were all negative. Lactate was within the normal range. cUS on admission showed abnormal echogenicity of both thalami (-Fig. 3A).
An MRI confirmed symmetrical thalamic lesions suggestive of acute hypoxic-ischemic injury (-Fig. 3B).

The MRI was repeated at term equivalent age and showed symmetrical thalamic lesions, lack of myelination and signal intensity changes in the white matter (-Fig. 3C).

Genetic testing was done because of the multiple congenital anomalies. A duplication of $210 \mathrm{~kb}$ of chromosome $4 q 35.1 q 35.2$ was found. This duplication was not considered to be the cause of his problems. Ophthalmological examination revealed no eye abnormalities.

The hearing screening test (AABR) was abnormal and a BERA was performed which showed a bilateral, mixed hearing loss of 0 to $30 \mathrm{~dB}$, with abnormal auditive processing of the brainstem. 


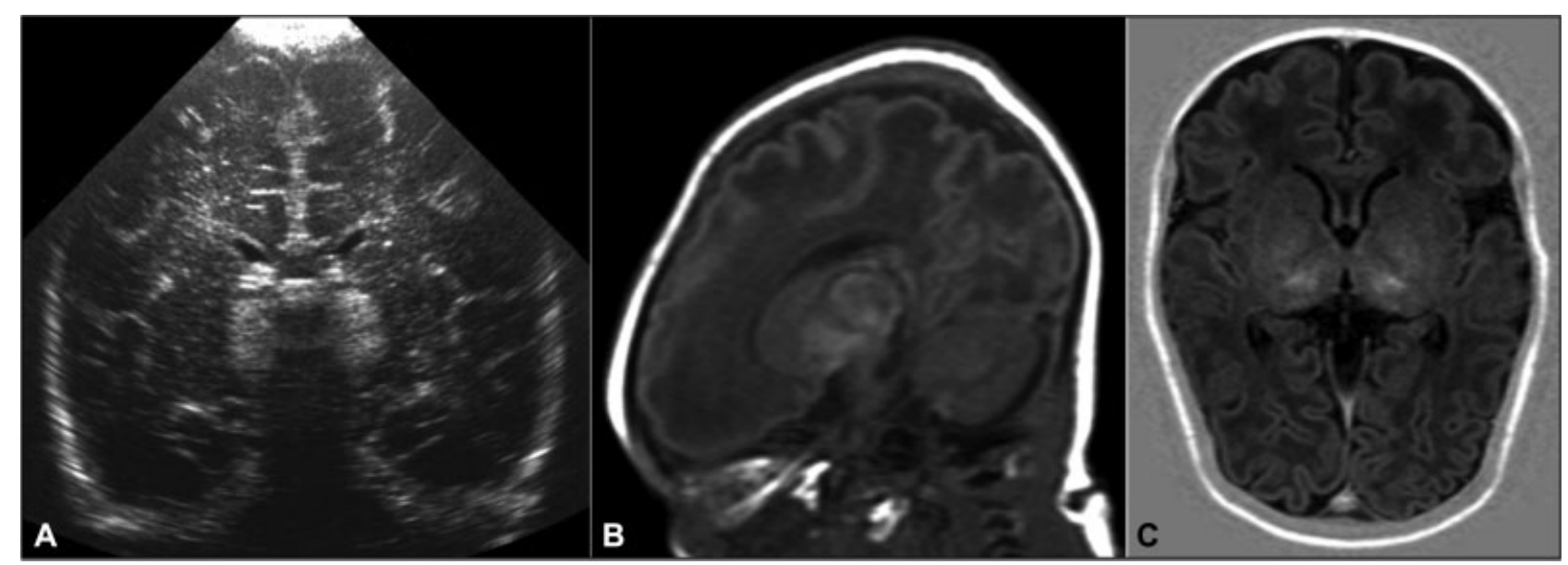

Fig. 3 Case 2. (A) Increased echogenicity of both thalami on cUS on admission. (B) MRI, sagittal T1-weighted sequence, confirmed symmetrical thalamic lesions. (C) MRI, axial T1-weighted image at term equivalent age showed increased signal intensity in both thalami, lack of myelination of the posterior limb of the internal capsule and reduced thalamic volumes. cUS, cranial ultrasound; MRI, magnetic resonance imaging.

At 6 weeks of age his general movements showed lack of variation and there was increased tone of his upper and lower limbs. He was discharged home, being tube fed and died at 2.5 months of age. No permission for postmortem examination was obtained.

\section{Discussion}

In neonates with symmetrical abnormalities in the thalami and without a clear history of perinatal asphyxia the diagnosis STL should be considered. Clinical symptoms are present at birth and cUS abnormalities may be present at birth but it may also take some time before these are clearly recognized. The echogenicity tends to increase over time and is mainly present in both thalami while less or even absent in the basal ganglia. It may be difficult to distinguish between hemorrhage and hypoxic-ischemic injury and MRI will contribute to making this distinction. ${ }^{7}$ Unlike thalamic injury in HIE, the abnormalities in the thalami are more prominent than in the basal ganglia and best seen on cUS. This is probably related to calcification in that area which is readily detected by cUS. In addition, seizures and (a)EEG abnormalities are rare because the major lesions are located deep in the brain, the cortex is usually unaffected and the acute event happened days or weeks before birth.

In preterm neonates the symmetrical thalamic echogenicity should not be confused with physiological echogenicity of the deep gray matter as is often seen in preterm neonates before term equivalent age. ${ }^{8}$ This physiological echogenicity is more diffuse and subtler and mainly located in the basal ganglia.

Conventional (T1 - and T2-weighted) MRI, performed at or after term equivalent age will show absent or delayed myelination of the posterior limb of the internal capsule. Signal intensity changes in the thalami, basal ganglia, and/or brainstem may be seen and are more outspoken on T1- than on T2-weighted images. ${ }^{9,10}$ In our case series 4 patients had additional abnormalities in the white matter (mainly punc- tate white matter lesions) and/or cerebellum (mainly small hemorrhages). CT may be helpful in showing the mineralization in the thalami, but is not recommended in neonates because of the radiation hazard. In addition, unless it is done in a unit with a lot of experience in neonatal CT, it will not improve the detection of calcification as compared with cUS.

Clinical symptoms in the neonatal period consist of abnormal tone (mostly hypertonia, sometimes preceded by hypotonia), absent sucking and swallowing reflexes, often facial diplegia and sometimes seizures. A prenatal cause for STL is considered most likely because of the abnormal tone, often associated with contractures and swallowing difficulties already present at birth or soon thereafter, and the cUS abnormalities that are striking at or soon after birth. The time needed to develop tissue calcification after a hypoxicischemic event is approximately 1 week and it will take at least this time period for the cUS abnormalities to evolve to their maximum extent. ${ }^{11}$

The selective vulnerability of the thalamus and basal ganglia for a severe hypoxic-ischemic event has been documented clinically as well as experimentally. ${ }^{12-14}$

In about one-third of all the STL-cases described so far there was polyhydramnios, a sign of abnormal fetal swallowing. In many cases, there was a severe antenatal event, probably leading to acute and severe fetal hypoxia and/or ischemia. Possible other causes of STL may be genetic, infectious, metabolic, and toxic. We found a case report in the literature of two siblings with STL, suggestive of a possible mutant autosomal recessive gene in both parents. ${ }^{15}$ We found no cases in the literature describing an infectious or a metabolic cause. We found two cases of a toxic cause, one of them was a salicylate-intoxication. ${ }^{16}$

In our cases, we found no evidence for a toxic, metabolic, or infectious cause. Two patients had a genetic defect (a small deletion on single nucleotide polymorphism [SNP] array in one and a short duplication in another one), but these were considered unlikely explanations for the STL seen in these patients. A recent case series, using next generation 
sequencing techniques (whole exome sequencing, WES) was unable to identify a genetic defect in three patients with STL. ${ }^{17}$

In all our cases, the most likely cause was severe antenatal hypoxia-ischemia, supported by a clear history in nine infants.

There was a striking female preponderance in our caseseries, but we did not find this in the existing literature. Hearing loss was present in six of our cases and has previously been described in the literature, but it is not a consistent finding. ${ }^{18}$

The prognosis of STL is very poor. Most children die within the 1 st year of life. The cause of death is usually aspiration pneumonia or severe apnea. So far, only one case has been reported who survived beyond 3 years of age. In our case series, two children survived beyond 3 years of age: case 1 , described above and case 5 who had severe developmental delay and developed spastic quadriplegia, GMFCS level III.

On histopathology bilateral lesions of the thalamus, mostly the lateral part, with gliosis, neuronal mineralization, and loss of neurons have been reported. ${ }^{11,16}$ There may be some histopathological overlap with Möebius's syndrome. ${ }^{6}$ This is in agreement with our autopsy data.

\section{Conclusion}

In infants with progressive symmetrical increased echogenicity in the thalami on cUS, STL should be considered. STL diagnosed in the neonatal period carries a poor prognosis. A thorough pregnancy history is needed to identify an acute antenatal insult which is the most likely underlying etiology.

Note

A statement of the location where the work was performed: this work has been performed at the NICU of Isala Women and Children's Hospital in close collaboration with two other European institutions.

\section{Conflict of Interest}

None.

\section{Acknowledgment}

We want to thank professor Frances Cowan (Imperial college of medicine, London, U.K.) for her contribution to the diagnosis in case 1.

\section{References}

1 Rosales RK, Riggs HE. Symmetrical thalamic degeneration in infants. J Neuropathol Exp Neurol 1962;21:372-376

2 Peters B, Walka MM, Friedmann W, Stoltenburg-Didinger G, Obladen M. Hypoxic-ischemic encephalopathy with cystic brain stem necroses and thalamic calcifications in a preterm twin. Brain Dev 2000;22(04):265-271

3 Buldini B, Drigo P, Via LD, Calderone M, Laverda AM. Symmetrical thalamic calcifications in a monozygotic twin: case report and literature review. Brain Dev 2005;27(01):66-69

4 Bordarier C, Moktari M, Rodriguez D, Adamsbaum C, Robain O. Antenatal thalamic lesions in the newborn: two anatomoclinical cases. Brain Dev 1997;19(07):495-498

5 Counsell SJ, Maalouf EF, Fletcher AM, et al. MR imaging assessment of myelination in the very preterm brain. AJNR Am J Neuroradiol 2002;23(05):872-881

6 Peleg D, Nelson GM, Williamson RA, Widness JA. Expanded Möbius syndrome. Pediatr Neurol 2001;24(04):306-309

7 Logitharajah P, Rutherford MA, Cowan FM. Hypoxic-ischemic encephalopathy in preterm infants: antecedent factors, brain imaging, and outcome. Pediatr Res 2009;66(02):222-229

8 van Wezel-Meijler G, Leijser LM, Wiggers-de Bruïne FT, Steggerda SJ, van der Grond J, Walther FJ. Diffuse hyperechogenicity of basal ganglia and thalami in preterm neonates: a physiologic finding? Radiology 2011;258(03):944-950

9 Roland EH, Poskitt K, Rodriguez E, Lupton BA, Hill A. Perinatal hypoxic-ischemic thalamic injury: clinical features and neuroimaging. Ann Neurol 1998;44(02):161-166

10 Natsume J, Watanabe K, Kuno K, Hayakawa F, Hashizume Y. Clinical, neurophysiologic, and neuropathological features of an infant with brain damage of total asphyxia type (Myers). Pediatr Neurol 1995; 13(01):61-64

11 Eicke M, Briner J, Willi U, Uehlinger J, Boltshauser E. Symmetrical thalamic lesions in infants. Arch Dis Child 1992;67(1, Spec No):15-19

12 Leech RW, Alvord EC Jr. Anoxic-ischemic encephalopathy in the human neonatal period. The significance of brain stem involvement. Arch Neurol 1977;34(02):109-113

13 Myers RE. Four patterns of perinatal brain damage and their conditions of occurrence in primates. Adv Neurol 1975;10:223-234

14 Parisi JE, Collins GH, Kim RC, Crosley CJ. Prenatal symmetrical thalamic degeneration with flexion spasticity at birth. Ann Neurol 1983;13(01):94-97

15 Abuelo DN, Barsel-Bowers G, Tutschka BG, Ambler M, Singer DB. Symmetrical infantile thalamic degeneration in two sibs. J Med Genet 1981;18(06):448-450

16 DiMario FJ Jr, Clancy R. Symmetrical thalamic degeneration with calcifications of infancy. Am J Dis Child 1989;143(09):1056-1060

17 Gorman KM, Aird JJ, Conroy J, Devaney D, Farrell M, King MD. Symmetrical thalamic calcification: A trio whole exome sequencing negative series. Brain Dev 2017;39(05):426-430

18 Banerjea MC, Speer CP. Bilateral thalamic lesions in a newborn with intrauterine asphyxia after maternal cardiac arrest-a case report with literature review. J Perinatol 2001;21(06):405-409 